BNL-NT-01/20

RBRC-195

August 2001

\title{
Next-to-leading order QCD evolution of transversity fragmentation functions
}

\author{
Marco Stratmann ${ }^{a}$ and Werner Vogelsang ${ }^{b, c}$ \\ ${ }^{a}$ Institut für Theoretische Physik, Universität Regensburg, \\ D-93040 Regensburg, Germany \\ ${ }^{b}$ Physics Department, Brookhaven National Laboratory, \\ Upton, New York 11973, U.S.A. \\ ${ }^{c}$ RIKEN-BNL Research Center, Bldg. 510a, Brookhaven National Laboratory, \\ Upton, New York 11973 - 5000, U.S.A.
}

\begin{abstract}
We derive the next-to-leading order splitting kernels for the scale evolution of fragmentation functions for transversely polarized quarks into transversely polarized hadrons.
\end{abstract}


Very little is known so far about spin effects in fragmentation of partons into hadrons. An exception is semi-inclusive production of $\Lambda$ baryons in $e^{+} e^{-}, e p$, or $p p$ scattering, where the polarization of the $\Lambda$ has been observed [1-4] and been related to corresponding spin-dependent QCD parton-to- $\Lambda$ fragmentation functions [5-7].

Polarization effects in fragmentation are by themselves interesting since their study opens up a new perspective on non-perturbative QCD phenomena in hadron formation. In addition it has been realized that, if known with sufficient accuracy, spin-dependent fragmentation functions can be used as "polarimeters" for nucleon structure [8-11]. For instance, if the polarized fragmentation functions for a transversely polarized quark producing a transversely polarized $\Lambda$ have been extracted accurately in $e^{+} e^{-}$annihilation, one can determine with their help the much coveted nucleon's transversity densities by studying two spin asymmetries with transverse polarization in $\Lambda$ production in ep or $p p$ collisions. Behind such a reasoning is of course the QCD factorization theorem which states that, for a given produced hadron, the fragmentation functions appearing in these scatterings are universal, provided of course the process is amenable at all to a description in terms of fragmentation functions. This is the case in the presence of a hard scale in the reaction under consideration, such as the virtuality $Q$ of the virtual boson in $e^{+} e^{-}$annihilation or the transverse momentum of the $\Lambda$ in $p p \rightarrow \Lambda X$.

It is expected that future experiments will give very precise information on spin-dependent fragmentation functions [12-14]. To analyze such data, an advanced theoretical framework is required. According to the factorization theorem the polarized cross section for, say, $\Lambda$ production in $p p^{\uparrow} \rightarrow \Lambda^{\uparrow} X$ (the arrow denoting transverse polarization) is given by a convolution of the form

$$
\begin{aligned}
\frac{E d \delta \sigma}{d p^{3}} & \equiv \frac{1}{2}\left[\frac{E d \sigma}{d p^{3}}(\uparrow \uparrow)-\frac{E d \sigma}{d p^{3}}(\uparrow \downarrow)\right] \\
& =\sum_{a, b, c} \int d x_{a} \int d x_{b} \int d z_{c} f_{a}\left(x_{a}, \mu\right) \delta f_{b}\left(x_{b}, \mu\right) \frac{E d \delta \hat{\sigma}_{a b}^{c}}{d p^{3}}(\hat{s}, \hat{t}, \hat{u}, \mu) \delta D_{c}^{\Lambda}\left(z_{c}, \mu\right)
\end{aligned}
$$

where $E, p$ are the energy and momentum of the produced $\Lambda$. It is again assumed that the reaction is characterized by a hard scale such as the transverse momentum $p_{T}$ of the $\Lambda$. We denote by $\hat{s}, \hat{t}, \hat{u}$ the Mandelstam variables for the partonic hard-scattering process $a b \rightarrow c X$. In Eq. (1), $f_{a}$ stands for the unpolarized distribution function of parton $a$, while the $\delta f_{b}$ are the transversity densities. The $\delta D_{c}^{H}(z, \mu)$ represent the transversity fragmentation functions, defined in analogy with the transversity distribution functions as

$$
\delta D_{c}^{\Lambda}(z, \mu) \equiv D_{c(\uparrow)}^{\Lambda(\uparrow)}(z, \mu)-D_{c(\uparrow)}^{\Lambda(\downarrow)}(z, \mu)
$$

where $D_{c(\uparrow)}^{\Lambda(\uparrow)}(z, \mu)\left(D_{c(\downarrow)}^{\Lambda(\uparrow)}(z, \mu)\right)$ denotes the probability for the fragmentation of a transversely 
polarized parton $c$ to a $\Lambda$ with aligned (anti-aligned) transverse spin, carrying the fraction $z$ of the parent parton's momentum. Finally, the sum in Eq. (1) is over all contributing partonic channels $a+b \rightarrow c+X$, with $E d \delta \hat{\sigma}_{a b}^{c} / d p^{3}$ the associated partonic cross section, defined in complete analogy with the first line of Eq. (1), the transverse polarizations now referring to partonic ones:

$$
\frac{E d \delta \hat{\sigma}_{a b}^{c}}{d p^{3}} \equiv \frac{1}{2}\left[\frac{E d \delta \hat{\sigma}_{a b}^{c}}{d p^{3}}(\uparrow \uparrow)-\frac{E d \delta \hat{\sigma}_{a b}^{c}}{d p^{3}}(\uparrow \downarrow)\right] .
$$

The $E d \delta \hat{\sigma}_{a b}^{c} / d p^{3}$ are perturbatively calculable thanks to the hard scale involved.

The factorized form of Eq. (11) implies the introduction of a scale $\mu \sim \mathcal{O}\left(p_{T}\right)$, the factorization scale, that reflects the certain amount of arbitrariness in the separation of short-distance and long-distance physics embodied in Eq. (11). Even though the parton densities and fragmentation functions cannot presently be derived from first principles, their dependence on $\mu$ is calculable perturbatively in terms of the "DGLAP" evolution equations [15], allowing to relate their values at one scale to their values at any other $\mu$. Dependence on $\mu$ also arises in the procedure of renormalizing the strong coupling constant. Note that in principle one could distinguish between factorization scales for the initial and final states and keep also the renormalization scale separate; however, for simplicity we keep all scales the same.

In practice, the precision of the framework of Eq. (1) largely depends on the perturbative order to which its ingredients are calculated. As already pointed out, there are two places where perturbation theory enters: first, the $E d \delta \hat{\sigma}_{a b}^{c} / d p^{3}$ have the expansion

$$
d \delta \hat{\sigma}_{a b}^{c}=d \delta \hat{\sigma}_{a b}^{c,(0)}+\left(\frac{\alpha_{s}}{\pi}\right) d \delta \hat{\sigma}_{a b}^{c,(1)}+\left(\frac{\alpha_{s}}{\pi}\right)^{2} d \delta \hat{\sigma}_{a b}^{c,(2)}+\ldots
$$

We note that currently partonic cross sections involving transverse polarization in the final state are only known at lowest order, except for the reactions $e^{+} e^{-} \rightarrow q^{\uparrow} \bar{q}^{\uparrow} X$ and $e q^{\uparrow} \rightarrow e q^{\uparrow} X$ for which first-order corrections have been calculated [16].

Secondly, the kernels governing the $\mu$-evolution of the parton densities and fragmentation functions also enjoy a perturbative expansion. In this paper we present the first-order corrections to the evolution of the transversity fragmentation functions defined in Eq. (2). This seems timely in view of forthcoming new experimental information on the production of transversely polarized $\Lambda$ 's [12-14]. In addition, the same evolution kernels [17 drive the evolution of the so-called interference fragmentation functions introduced in Ref. [10].

An important observation concerning the $\delta D_{c}^{\Lambda}$ is that at leading power in the hard scale there is no gluonic transversity fragmentation function $\delta D_{g}^{\Lambda}$ due to angular momentum conservation and the helicity-flip nature of the $\delta D_{c}^{\Lambda}[18-20]$. This feature also implies that there is no 
mixing with gluons in the $\mu$-evolution of the $\delta D_{q}^{\Lambda}, \delta D_{\bar{q}}^{\Lambda}$. The evolution equations are then most conveniently written in terms of the combinations

$$
\delta D_{q, \pm}^{\Lambda} \equiv \delta D_{q}^{\Lambda} \pm \delta D_{\bar{q}}^{\Lambda}
$$

for which they read:

$$
\frac{d}{d \ln \mu^{2}} \delta D_{q, \pm}^{\Lambda}(z, \mu)=\int_{z}^{1} \frac{d y}{y} \delta P_{q q, \pm}^{(T)}\left(y, \alpha_{s}(\mu)\right) \delta D_{q, \pm}^{\Lambda}\left(\frac{z}{y}, \mu\right)
$$

Here the superscript " $(T)$ " stands for "time-like" and indicates that we are dealing with a fragmentation function. It is instructive to confront Eq. (6) with the corresponding evolution equations for the ("space-like") transversity distribution functions [21, 18, 19, 20] in, say, the proton, $\delta q_{ \pm} \equiv \delta q \pm \delta \bar{q}$ :

$$
\frac{d}{d \ln \mu^{2}} \delta q_{ \pm}(x, \mu)=\int_{x}^{1} \frac{d y}{y} \delta P_{q q, \pm}^{(S)}\left(y, \alpha_{s}(\mu)\right) \delta q_{ \pm}\left(\frac{x}{y}, \mu\right) .
$$

The evolution kernels $\delta P_{q q, \pm}^{(U)}(U=T, S)$ occurring in Eqs. (6), (7) have the perturbative expansion

$$
\delta P_{q q, \pm}^{(U)}\left(\xi, \alpha_{s}\right)=\left(\frac{\alpha_{s}}{2 \pi}\right) \delta P_{q q, \pm}^{(U),(0)}(\xi)+\left(\frac{\alpha_{s}}{2 \pi}\right)^{2} \delta P_{q q, \pm}^{(U),(1)}(\xi)+\ldots
$$

They are in general not identical, but are closely related to each other, as can be inferred from studies of fragmentation functions in the unpolarized case or in the case of longitudinal polarization [22-25]. To lowest order, the space-like and time-like splitting functions actually do agree, and there is also no distinction between the kernels for the evolution of the + and combinations of densities:

$$
\delta P_{q q, \pm}^{(T),(0)}(\xi) \equiv \delta P_{q q, \pm}^{(S),(0)}(\xi)=C_{F}\left[\frac{2 \xi}{(1-\xi)_{+}}+\frac{3}{2} \delta(1-\xi)\right]
$$

where $C_{F}=4 / 3$, and the + -prescription is defined in the usual way by

$$
\int_{0}^{1} d z f(z)[g(z)]_{+} \equiv \int_{0}^{1} d z[f(z)-f(1)] g(z) .
$$

The splitting function $\delta P_{q q, \pm}^{(S),(0)}$ was derived in [26, 20, 27]. The identity of $\delta P_{q q, \pm}^{(T),(0)}$ and $\delta P_{q q, \pm}^{(S),(0)}$ is a manifestation of the so-called Gribov-Lipatov relation (GLR) [28] which connects spacelike and time-like structure functions within their respective physical regions $(\xi<1)$. The space-like and time-like leading order splitting functions are also directly related by analytic continuation through $x=1$ :

$$
\begin{aligned}
\delta P_{q q, \pm}^{(T),(0)}(z) & =-z \delta P_{q q, \pm}^{(S),(0)}\left(\frac{1}{z}\right) \\
& \equiv \mathcal{A C}\left[\delta P_{q q, \pm}^{(S),(0)}(x)\right]
\end{aligned}
$$


where $x=1 / z$ and $z<1$. Eq. (11) represents the analytic continuation or Drell-Levy-Yan relation $(\mathrm{ACR})$ 29]. In the second line we have introduced the operation $\mathcal{A C}$ that analytically continues any space-like function to $x \rightarrow 1 / z>1$ and correctly adjusts the normalization. Note that the endpoint contributions $\propto \delta(1-z)$ are not subject to the $\mathcal{A C}$ operation; however, they are necessarily identical in the space-like and time-like cases.

Both the GLR and the ACR are known to be violated beyond the lowest order [22, 24, 25]. However, the ACR is based on symmetries of tree diagrams under crossing, and therefore its breaking at next-to-leading order (NLO) is essentially of kinematical origin within a given regularization prescription, as was shown in Ref. [24]. The main issue here is an extra factor of $z^{-2 \epsilon}$ (in $\overline{\mathrm{MS}}$-scheme dimensional regularization with $n=4-2 \epsilon$ space-time dimensions) in phase space for the time-like case which, when combined with terms singular at $\epsilon=0$, generates extra terms $\propto \ln z$ in the final answer of the time-like NLO splitting functions. It is then fairly straightforward to go through the calculation of Ref. [30] of the space-like NLO transversity splitting functions, and to identify in each contributing Feynman diagram the ACR breaking effects in the procedure of analytic continuation to $x>1$.

Combining all extra terms, we obtain in the $\overline{\mathrm{MS}}$ scheme:

$$
\delta P_{q q, \pm}^{(T),(1)}(z)=\mathcal{A C}\left[\delta P_{q q, \pm}^{(S),(1)}(x)\right]+\beta_{0} \delta P_{q q, \pm}^{(S),(0)}(z) \ln z
$$

(for $z<1$ ) where $\beta_{0}=11 / 3 C_{A}-4 / 3 T_{R} n_{f}$ with $C_{A}=3, T_{R}=1 / 2$, and the number of active flavors, $n_{f}$. The last term in Eq. (12) obviously represents the breaking of the ACR. It is worth pointing out that the structure of that term with its proportionality to both $\beta_{0}$ and the lowestorder splitting function is that of a typical factorization scheme transformation. In other words, we could choose a (non- $\overline{\mathrm{MS}}$ ) factorization scheme in which the time-like transversity splitting functions would be given by $\mathcal{A C}\left[\delta P_{q q, \pm}^{(S),(1)}(x)\right]$, without any extra term, so that no breaking of the ACR would occur. This possibility was first demonstrated for the unpolarized [31] and longitudinally polarized [24] cases, which are more general in the sense that singlet mixing is present there. In the following, we do stay within the more conventional $\overline{\mathrm{MS}}$ scheme, however.

Inserting the explicit result of [30] for the $\operatorname{NLO} \delta P_{q q, \pm}^{(S),(1)}(x)$, performing the analytic continuation, and adding the endpoint contributions, we arrive at the final result for $\delta P_{q q, \pm}^{(T),(1)}(z)$. We first define

$$
\begin{aligned}
\delta p_{q q}^{(0)}(z) & \equiv \frac{2 z}{(1-z)_{+}} \\
\mathcal{S}_{2}(z) & =\int_{\frac{z}{1+z}}^{\frac{1}{1+z}} \frac{d y}{y} \ln \left(\frac{1-y}{y}\right)
\end{aligned}
$$




$$
=-2 \operatorname{Li}_{2}(-z)-2 \ln z \ln (1+z)+\frac{1}{2} \ln ^{2} z-\frac{\pi^{2}}{6}
$$

where $\mathrm{Li}_{2}(z)$ is the dilogarithm function. Then

$$
\delta P_{q q, \pm}^{(T),(1)}(z) \equiv \delta P_{q q}^{(T),(1)}(z) \pm \delta P_{q \bar{q}}^{(T),(1)}(z)
$$

where

$$
\begin{aligned}
\delta P_{q q}^{(T),(1)}(z)= & C_{F}^{2}\left[1-z+\left(\frac{3}{2}+2 \ln (1-z)-2 \ln z\right) \ln z \delta p_{q q}^{(0)}(z)\right. \\
& \left.+\left(\frac{3}{8}-\frac{\pi^{2}}{2}+6 \zeta(3)\right) \delta(1-z)\right] \\
+ & \frac{1}{2} C_{F} C_{A}\left[-(1-z)+\left(\frac{67}{9}+\frac{11}{3} \ln z+\ln ^{2} z-\frac{\pi^{2}}{3}\right) \delta p_{q q}^{(0)}(z)\right. \\
& \left.+\left(\frac{17}{12}+\frac{11 \pi^{2}}{9}-6 \zeta(3)\right) \delta(1-z)\right] \\
+ & \frac{2}{3} C_{F} T_{R} n_{f}\left[\left(-\ln z-\frac{5}{3}\right) \delta p_{q q}^{(0)}(z)-\left(\frac{1}{4}+\frac{\pi^{2}}{3}\right) \delta(1-z)\right], \\
\delta P_{q \bar{q}}^{(T),(1)}(z)= & C_{F}\left(C_{F}-\frac{1}{2} C_{A}\right)\left[-(1-z)+2 \mathcal{S}_{2}(z) \delta p_{q q}^{(0)}(-z)\right],
\end{aligned}
$$

where $\zeta(3) \approx 1.202057$.

We are also in the position now to obtain the difference $\delta P_{q q, \pm}^{(T),(1)}(z)-\delta P_{q q, \pm}^{(S),(1)}(z)$ which is non-zero if the GLR is violated. Using Eqs. (16) and (17) along with the results of Ref. [30], we find:

$$
\delta P_{q q, \pm}^{(T),(1)}(z)-\delta P_{q q, \pm}^{(S),(1)}(z)=C_{F}^{2} \ln z \delta p_{q q}^{(0)}(z)(3+4 \ln (1-z)-2 \ln z)
$$

Note that as in the unpolarized and longitudinally polarized cases [22, 24] the violation of the GLR only occurs in the $C_{F}^{2}$ part of the splitting function.

For numerical applications it is convenient to have the Mellin- $n$ moments of the NLO splitting functions $\delta P_{q q, \pm}^{(T),(1)}(z)$, defined as

$$
\delta \tilde{P}_{q q, \eta}^{(T),(1)}(n) \equiv \int_{0}^{1} d z z^{n-1} \delta P_{q q, \pm}^{(T),(1)}(z)
$$

We obtain:

$$
\delta \tilde{P}_{q q, \eta}^{(T),(1)}(n)=\delta \tilde{P}_{q q, \eta}^{(S),(1)}(n)+2 C_{F}^{2}\left[4 S_{1}(n)-3\right]\left[\frac{\pi^{2}}{6}-S_{2}(n)\right]
$$




$$
\begin{aligned}
& =C_{F}^{2}\left[\frac{3}{8}+\frac{1-\eta}{n(n+1)}+3 S_{2}(n)-4 S_{1}(n)\left(3 S_{2}(n)-S_{2}^{\prime}\left(\frac{n}{2}\right)\right)-8 \tilde{S}(n)+S_{3}^{\prime}\left(\frac{n}{2}\right)\right. \\
& \left.\quad+\frac{\pi^{2}}{3}\left(4 S_{1}(n)-3\right)\right] \\
& +\frac{1}{2} C_{F} C_{A}\left[\frac{17}{12}-\frac{1-\eta}{n(n+1)}-\frac{134}{9} S_{1}(n)+\frac{22}{3} S_{2}(n)\right. \\
& \left.\quad+4 S_{1}(n)\left(2 S_{2}(n)-S_{2}^{\prime}\left(\frac{n}{2}\right)\right)+8 \tilde{S}(n)-S_{3}^{\prime}\left(\frac{n}{2}\right)\right] \\
& +\frac{2}{3} C_{F} T_{R} n_{f}\left[-\frac{1}{4}+\frac{10}{3} S_{1}(n)-2 S_{2}(n)\right],
\end{aligned}
$$

where $\eta \equiv \pm$, and where in the first line we have expressed the result in terms of the moments of the space-like NLO transversity splitting functions [30, 32, 33]. The sums appearing above are defined by

$$
\begin{aligned}
S_{k}(n) & \equiv \sum_{j=1}^{n} \frac{1}{j^{k}} \\
S_{k}^{\prime}\left(\frac{n}{2}\right) & \equiv 2^{k-1} \sum_{j=1}^{n} \frac{1+(-1)^{j}}{j^{k}} \\
\tilde{S}(n) & \equiv \sum_{j=1}^{n} \frac{(-1)^{j}}{j^{2}} S_{1}(j) .
\end{aligned}
$$

Their analytic continuations to arbitrary Mellin- $n$ (which depend on $\eta$ ) can be found in [34].

In summary, we have presented the next-to-leading order kernels for the evolution of transversity fragmentation functions. Our results will become useful in analyses of future precision data sensitive to leading-twist transverse-spin effects in fragmentation.

\section{Acknowledgments}

We are grateful to D. Boer for pointing out to us the relevance of this project and for helpful discussions. M.S. thanks RIKEN and Brookhaven National Laboratory for hospitality and support. W.V. is grateful to RIKEN, Brookhaven National Laboratory and the U.S. Department of Energy (contract number DE-AC02-98CH10886) for providing the facilities essential for the completion of this work. 


\section{References}

[1] D. Buskulic et al., ALEPH Collab., Phys. Lett. B374, 319 (1996);

K. Ackerstaff et al., OPAL Collab., Eur. Phys. J. C2, 49 (1998).

[2] A. Airapetian et al., HERMES Collab., hep-ex/9911017.

[3] M.R. Adams et al., E665 Collab., Eur. Phys. J. C17, 263 (2000).

[4] P. Astier et al., NOMAD Collab., Nucl. Phys. B588, 3 (2000).

[5] M. Burkardt and R.L. Jaffe, Phys. Rev. Lett. 70, 2537 (1993);

K. Chen, G. Goldstein, R.L. Jaffe, and X. Ji, Nucl. Phys. B445, 380 (1995).

[6] D. de Florian, M. Stratmann, and W. Vogelsang, Phys. Rev. D57, 5811 (1998).

[7] A. Kotzinian, A. Bravar, and D. von Harrach, Eur. Phys. J. C2, 329 (1998);

B.-Q. Ma and J. Soffer, Phys. Rev. Lett. 82, 2250 (1999);

B.-Q. Ma, J. Soffer, and J.-J. Yang, Phys. Rev. D61, 034017 (2000);

B.-Q. Ma, I. Schmidt, J. Soffer, and J.-J. Yang, Eur. Phys. J. C16, 657 (2000); Phys.

Lett. B488, 254 (2000); Phys. Rev. D62, 114009 (2000); ibid. D64, 014017 (2001);

hep-ph/0107157;

C. Boros, J.T. Londergan, and A.W. Thomas, Phys. Rev. D61, 014007 (2000); ibid. D62, 014021 (2000);

J.-J. Yang, Phys. Lett. B512, 57 (2001).

[8] J.C. Collins, Nucl. Phys. B396, 161 (1993);

J.C. Collins, S.F. Heppelmann, and G.A. Ladinsky, Nucl. Phys. B420, 565 (1994);

J.C. Collins and G.A. Ladinsky, hep-ph/9411444.

[9] X. Ji, Phys. Rev. D49, 114 (1994).

[10] R.L. Jaffe, X. Jin, and J. Tang, Phys. Rev. Lett. 80, 1166 (1998); Phys. Rev. D57, 5920 (1998).

[11] D. de Florian, J. Soffer, M. Stratmann, and W. Vogelsang, Phys. Lett. B439, 176 (1998).

[12] See, for example: G. Bunce, N. Saito, J. Soffer, and W. Vogelsang, Annu. Rev. Nucl. Part. Sci. 50, 525 (2000).

[13] HERMES Collab., "The HERMES Physics Program \& Plans for 2001-2006", DESY-PRC (2000);

G. Baum et al., COMPASS Collab., CERN/SPSLC 96-14 (1996). 
[14] A. Ogawa, talk given at the RIKEN BNL Research Center Workshop on "Spin Physics at RHIC in Year-1 and Beyond", BNL, May 2001, BNL report BNL-52635, p. 225.

[15] G. Altarelli and G. Parisi, Nucl. Phys. B126, 298 (1977);

Yu.L. Dokshitser, Sov. Phys. JETP 46, 641 (1977);

L.N. Lipatov, Sov. J. Nucl. Phys. 20, 95 (1975);

V.N. Gribov and L.N. Lipatov, Sov. J. Nucl. Phys. 15, 438 (1972).

[16] A.P. Contogouris, O. Korakianitis, Z. Merebashvili, and F. Lebessis, Phys. Lett. B344, 370 (1995);

A.P. Contogouris, O. Korakianitis, and Z. Merebashvili, Phys. Lett. B365, 334 (1996).

[17] D. Boer, talk presented at the "9th International Workshop on Deep Inelastic Scattering (DIS 2001)", Bologna, Italy, April 2001, hep-ph/0106206.

[18] R.L. Jaffe and X. Ji, Phys. Rev. Lett. 67, 552 (1991); Nucl. Phys. B375, 527 (1992).

[19] X. Ji, Phys. Lett. B289, 137 (1992).

[20] X. Artru and M. Mekhfi, Z. Phys. C45, 669 (1990).

[21] J.P. Ralston and D.E. Soper, Nucl. Phys. B152, 109 (1979);

J.L. Cortes, B. Pire, and J.P. Ralston, Z. Phys. C55, 409 (1992).

[22] G. Curci, W. Furmanski, and R. Petronzio, Nucl. Phys. B175, 27 (1980).

[23] W. Furmanski and R. Petronzio, Phys. Lett. 97B, 437 (1980).

[24] M. Stratmann and W. Vogelsang, Nucl. Phys. B496, 41 (1997).

[25] J. Blümlein, V. Ravindran, and W.L. van Neerven, Nucl. Phys. B586, 349 (2000).

[26] F. Baldracchini, N.S. Craigie, V. Roberto, and M. Socolovsky, Fortsch. Phys. 30, 505 (1981).

[27] J. Blümlein, Eur. Phys. J. C20, 683 (2001).

[28] V.N. Gribov and L.N. Lipatov, Sov. J. Nucl. Phys. 15, 438 (1972); ibid. 675 (1972).

[29] S.D. Drell, D.J. Levy, and T.M. Yan, Phys. Rev. 187, 2159 (1969); Phys. Rev. D1, 1617 (1970).

[30] W. Vogelsang, Phys. Rev. D57, 1886 (1998). 
[31] L. Beaulieu, E.G. Floratos, and C. Kounnas, Nucl. Phys. B166, 321 (1980).

[32] S. Kumano and M. Miyama, Phys. Rev. D56, 2504 (1997).

[33] A. Hayashigaki, Y. Kanazawa, and Y. Koike, Phys. Rev. D56, 7350 (1997).

[34] M. Glück, E. Reya, and A. Vogt, Z. Phys. C48, 471 (1990). 